\title{
RXTE Observation of Cygnus X-1: Spectra and Tìming
}

\section{J. Wilms' ${ }^{1}$ J. Dove' 2 , M. Nowak ${ }^{2}$, B. A. Vaughan 3}

${ }^{1}$ IAA Tübingen, Astronomic, Waldhäuser Str. 64, D-72076 Tübingen ${ }^{2}$ JILA, University of Colorado, Campus Box 440, Boulder, CO 80309-(0440

${ }^{3}$ Space Radiation Laboratory, Caltech, Pasadena, CA 91125

\section{Abstract}

We present first results from the analysis of an RXTE observation of Cyg X-1 in its low state, taken about two months after the end of the high state. With $\Gamma \approx 1.45$ the spectrum is considerably harder than previous lowstate measurements. The observed spectrum can be explained by a Comptonization spectrum as that emitted from a spherical corona surrounded by a cold accretion disk. The optical depth of the corona is between 2 and 2.5 and the temperature is between 60 and $80 \mathrm{keV}$. Temporal analysis shows a typical RMS noise of $\approx 25 \%$. The PSD can be described as consisting of a flat component followed by an $f^{-1}$ power-law, followed by an $f^{-1.6}$ power law. The lag of the hard photons with respect to the soft photons is consistent with prior observations. The coherence function is remarkably close to unity from $0.01 \mathrm{~Hz}$ to $10 \mathrm{~Hz}$. 


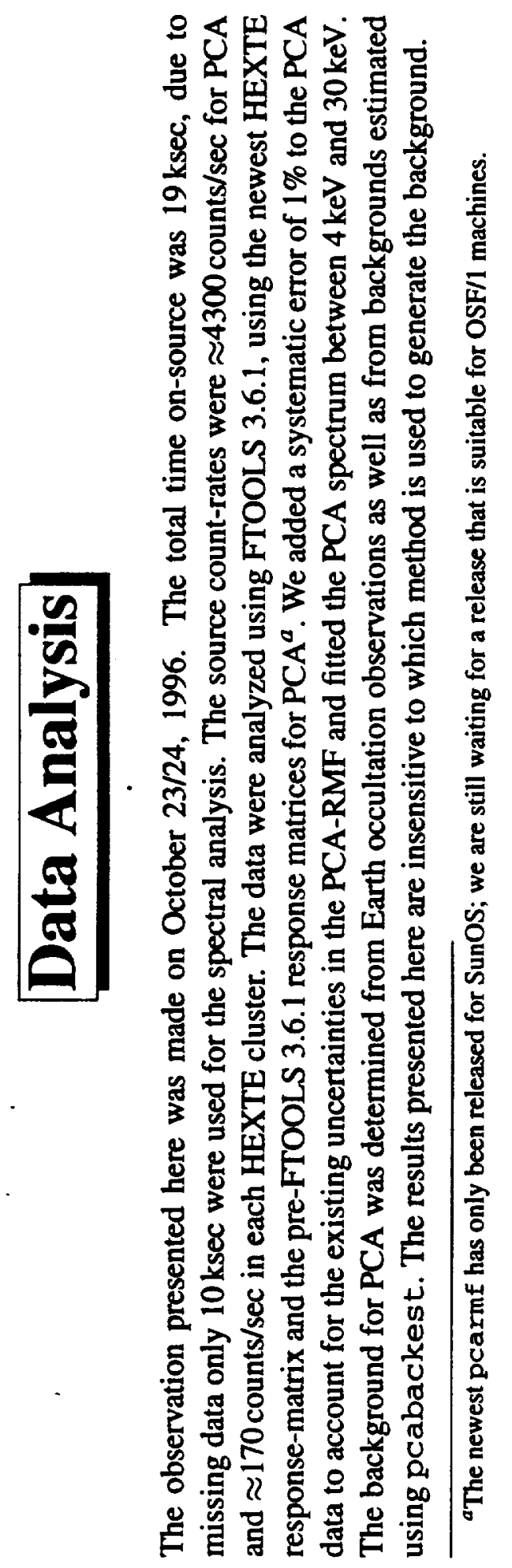




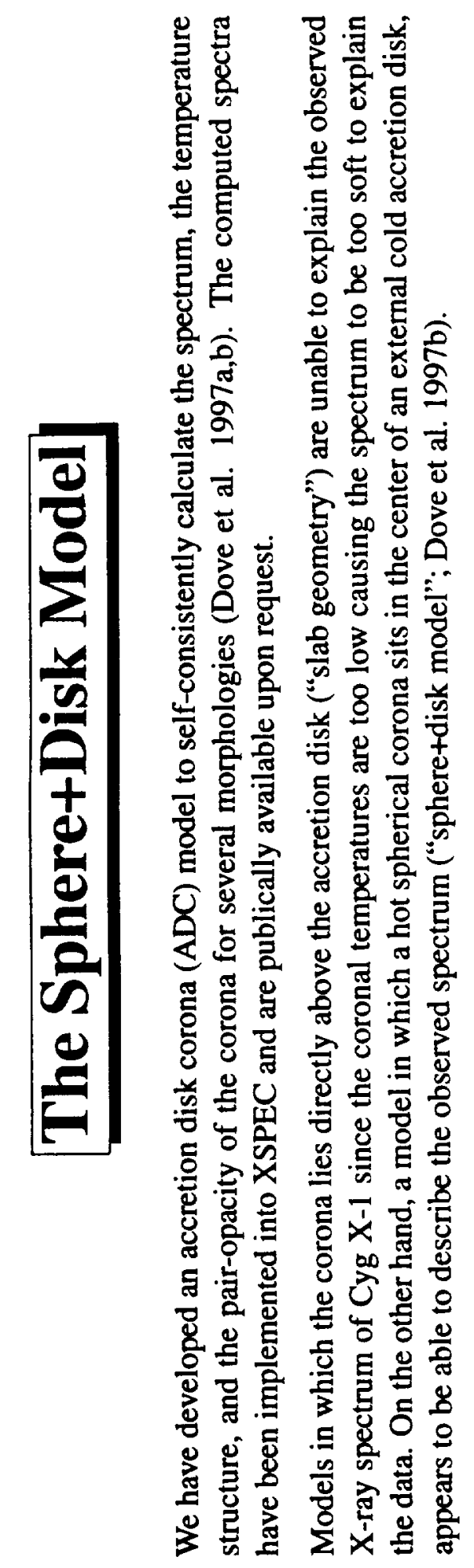




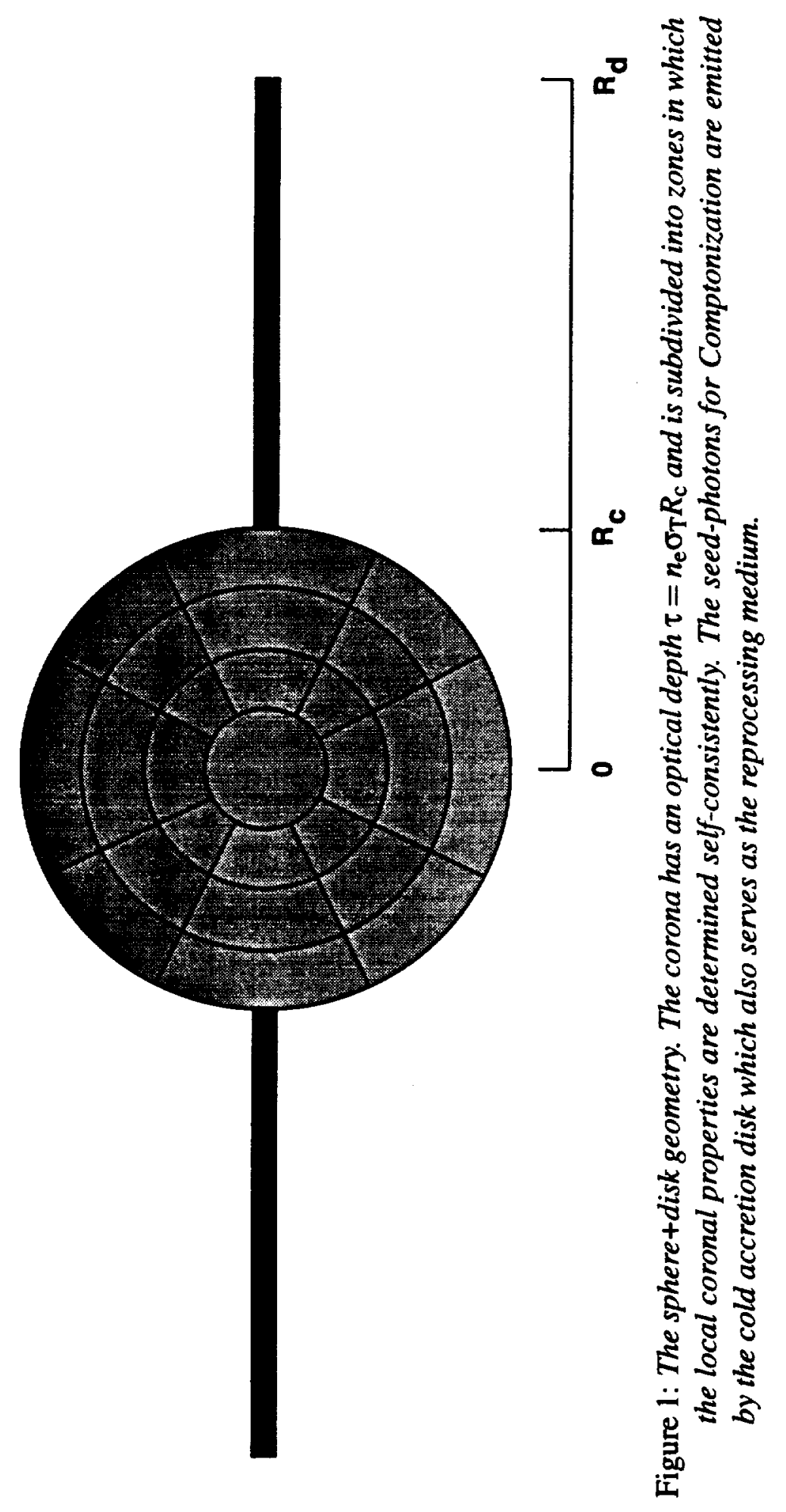




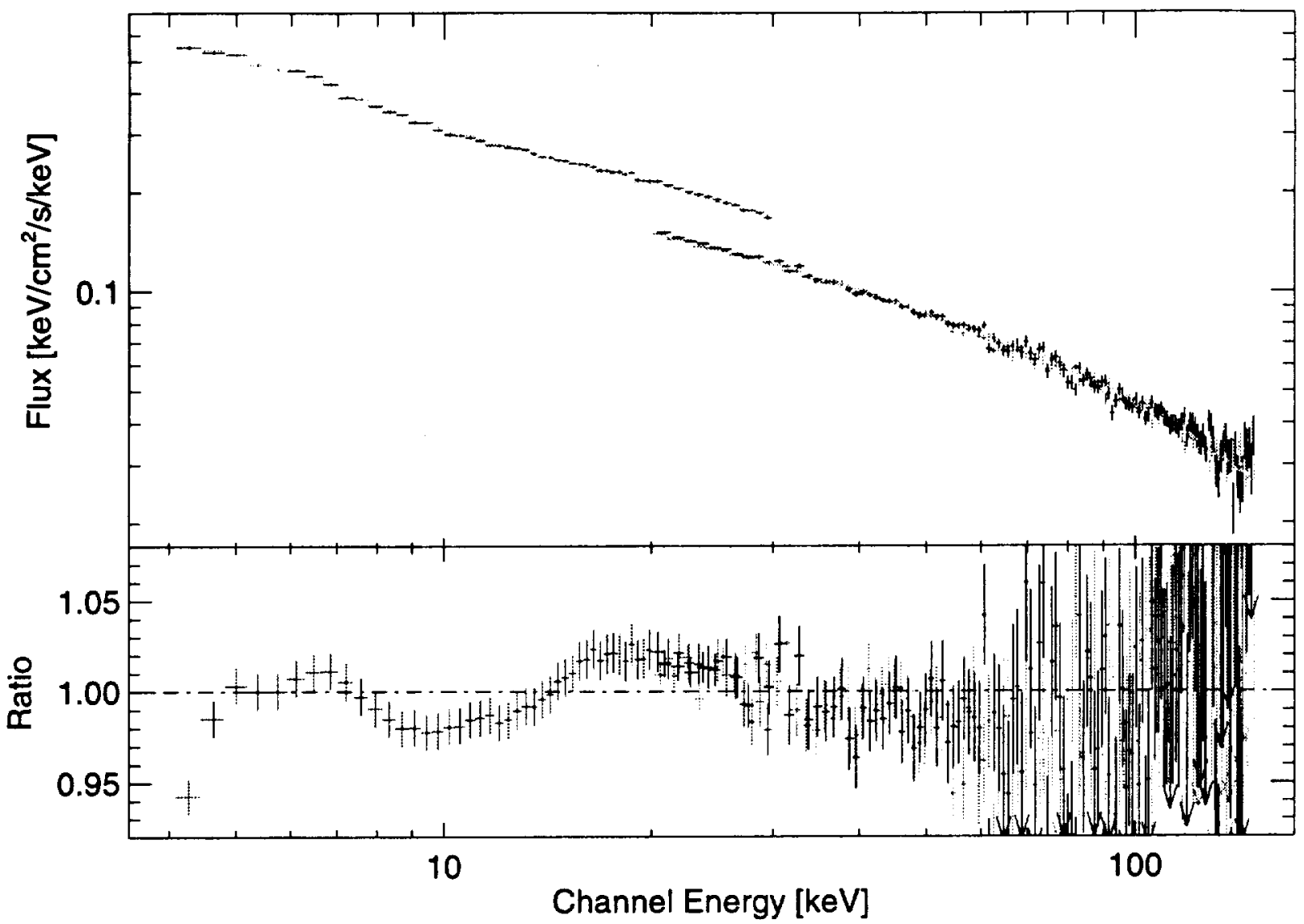

Figure 2: Comparison of the RXTE spectrum to the best-fit sphere+disk model. Here, the optical depth of the sphere is 2.6 and the temperature is $60 \mathrm{keV}$. The temperature of the accretion disk was assumed to be $T(r)=300 \mathrm{keV} \cdot r^{-0.75}$ where $r=\left(R / R_{\mathrm{c}}\right)($ see fig. 1$)$ and $N_{\mathrm{H}}$ was fixed at $6 \times 10^{21} \mathrm{~cm}^{-2}$. 


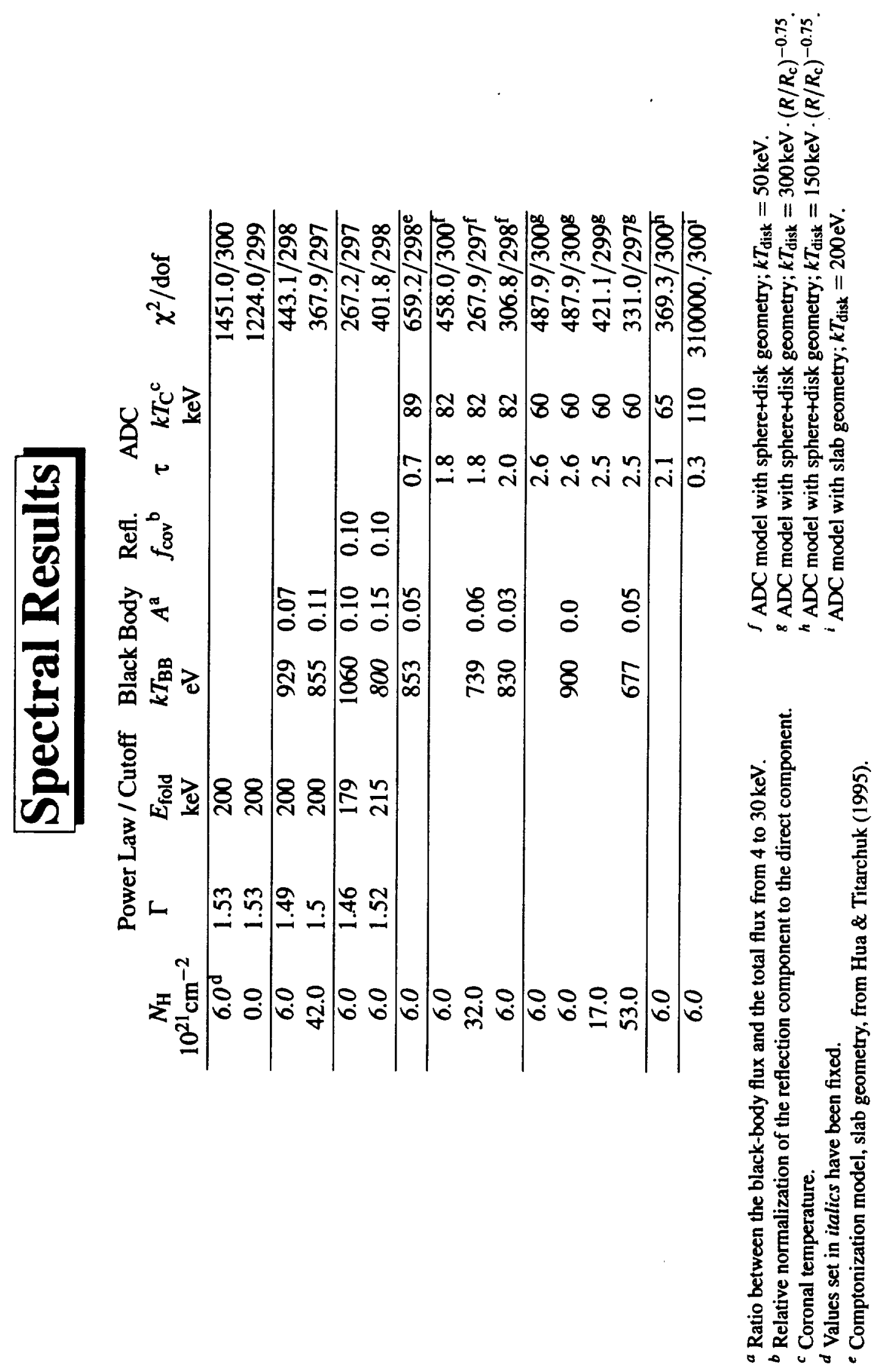




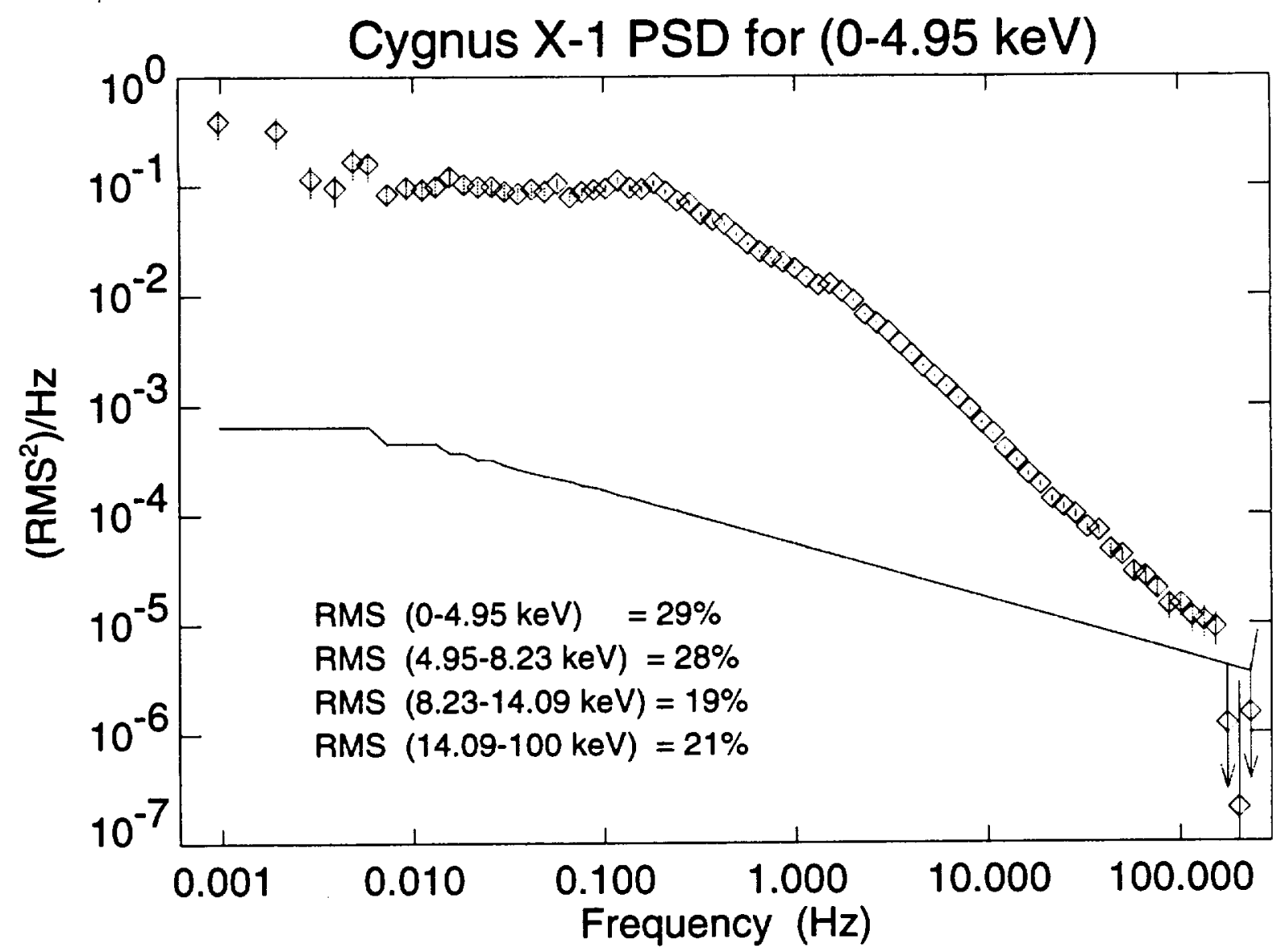

Figure 3: PSD for the energy-band below $5 \mathrm{keV}$ for a $10 \mathrm{ks}$ observation. The $\mathrm{mm}$-noise has been subtracted from the PSD, the sensitivity-level of which is indicated by the thin line. Normalization follows Miyamoto et al. (1992).

The PSD can be roughly described as

$$
\frac{\mathrm{PSD}}{\mathrm{rms}^{2} / \mathrm{Hz}}= \begin{cases}0.091 & \text { for } f<0.2 \mathrm{~Hz} \\ 0.017 f^{-1.04} & \text { for } 0.2 \mathrm{~Hz} \leq f<2.4 \mathrm{~Hz} \\ 0.031 f^{-1.71} & \text { for } 2.4 \mathrm{~Hz} \leq f\end{cases}
$$

where the break frequencies and power-law slopes have been determined numerically. 


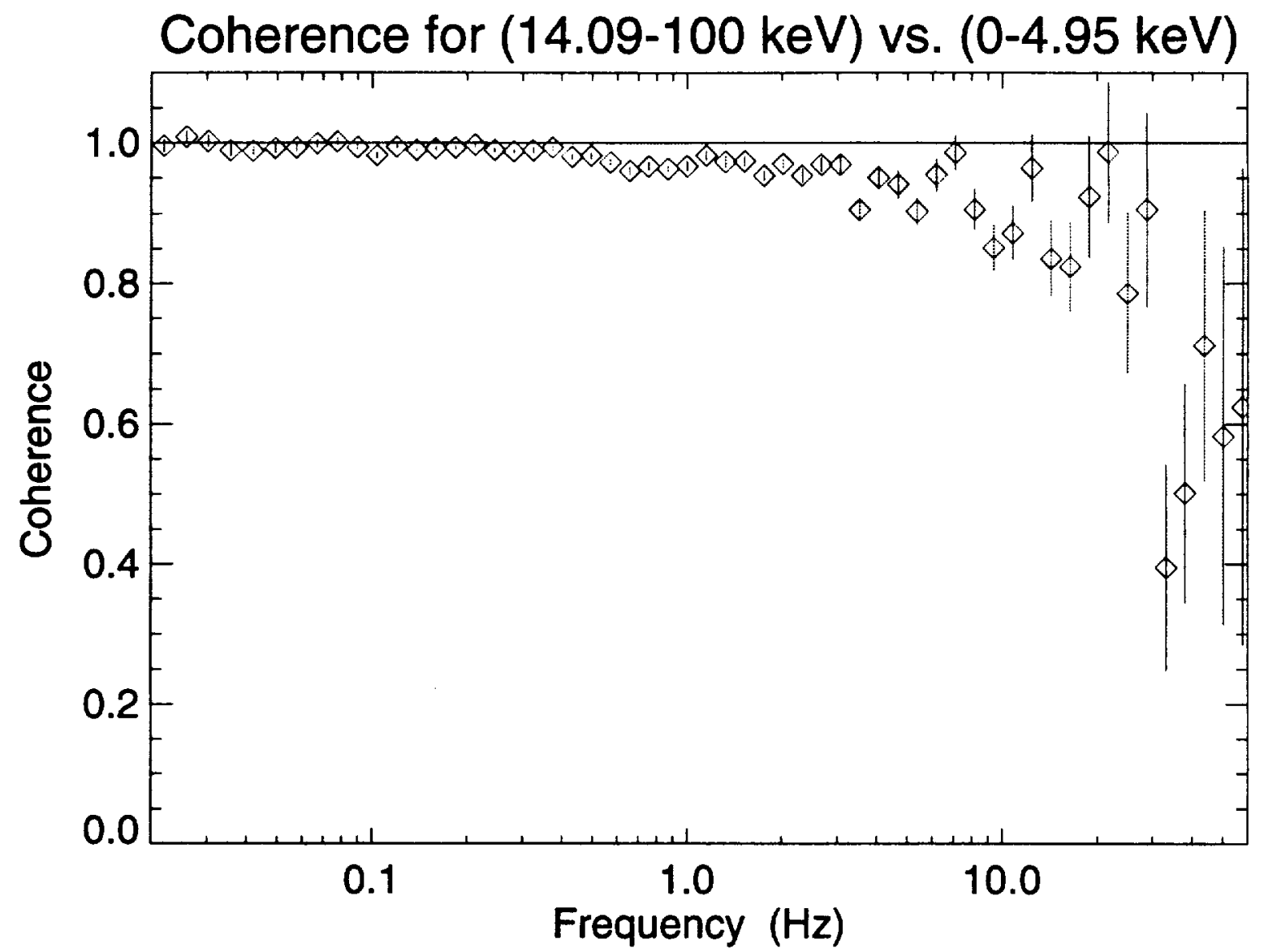

Figure 4: Coherence between the hard and soft energies.

The coherence function (Nowak \& Vaughan (1996); Vaughan \& Nowak (1997)) is defined as

$$
\gamma^{2}(f)=\frac{|<C(f)>|^{2}}{\left.\left.<\left|S_{1}(f)\right|^{2}\right\rangle<\left|S_{2}(f)\right|^{2}\right\rangle}
$$

where $C(f)$ is the cross-spectrum between the two observed signals $X_{i}(f)=S_{i}(f)+N_{i}(f)$, where $S_{i}(f)$ is the true signal and $N_{i}(f)$ is the Poisson noise-component ("observational rms noise"). The measured coherence of unity from $0.01 \mathrm{~Hz}$ to $10 \mathrm{~Hz}$ indicates a remarkable stability in the timing properties of the signal over the whole spectrum - an indication that either there is a single global source for the observed fluctuations, or that there is a global coherent response from the Compton corona, or both (Nowak et al. 1996). 


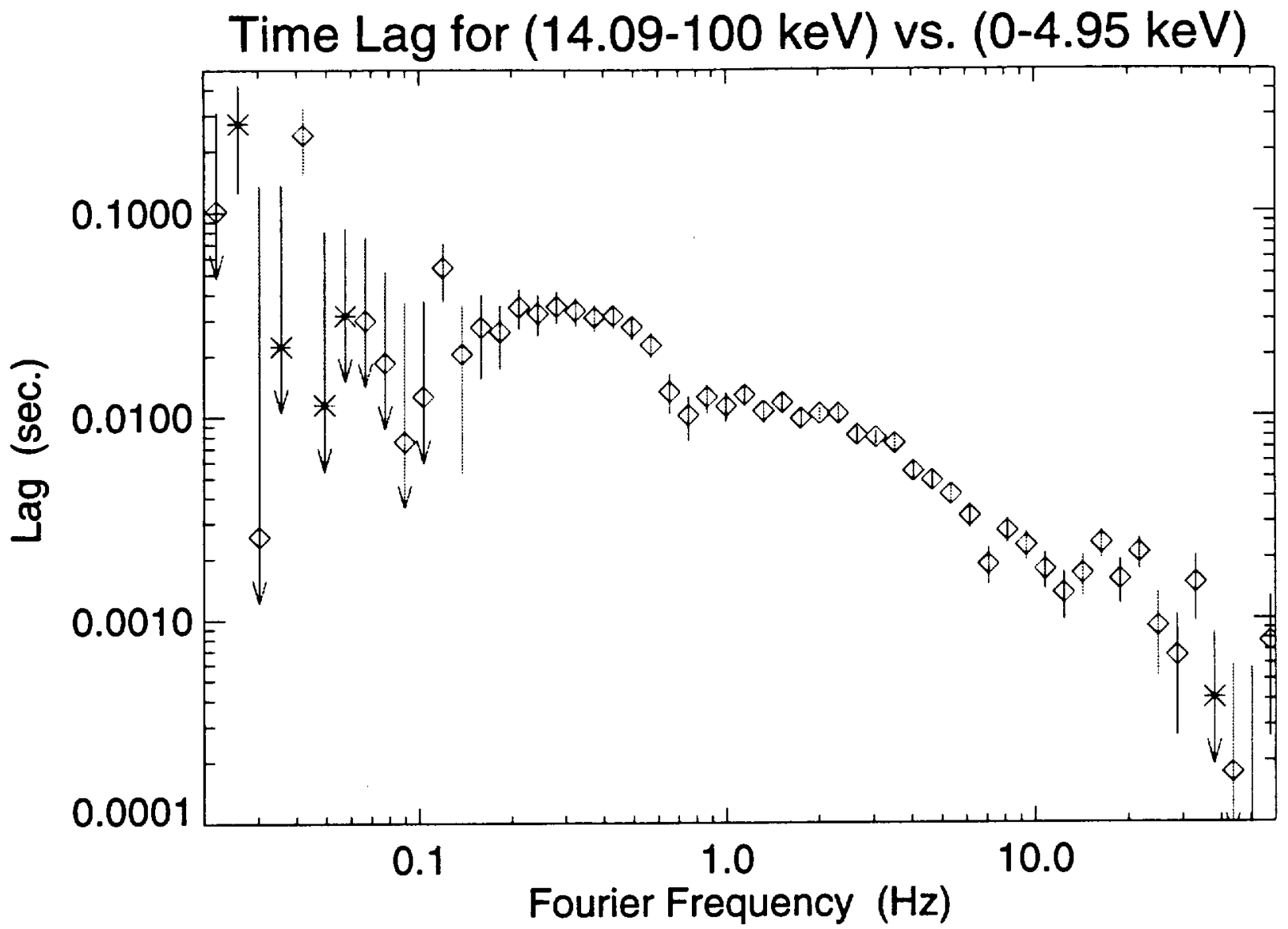

Figure 5: Time lag measured between the hard and the soft energy band. Red: the hard band lags the soft band, Blue: soft band lags the hard band.

The time-lag is well determined in the regime where the coherence function is unity, i.e., $0.01 \mathrm{~Hz}$ to $10 \mathrm{~Hz}$. Above $\approx 10 \mathrm{~Hz}$ the coherence is not preserved and therefore the time-lag varies erratically. The kinks at $0.8 \mathrm{~Hz}$ and $\approx 15 \mathrm{~Hz}$ are real (Miyamoto et al. 1992). 


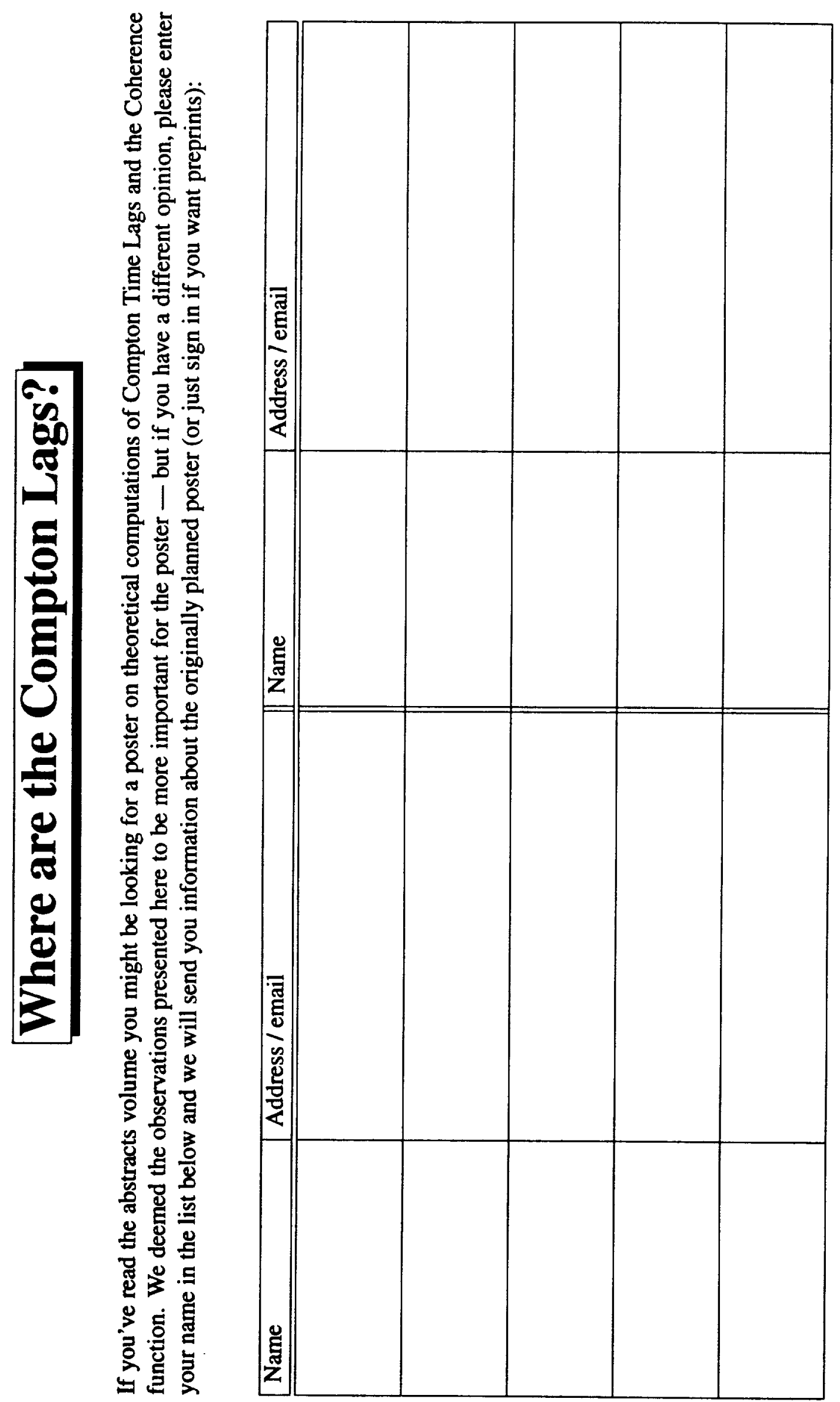




\section{References}

Dove, J. B., Wilms, J., Begelman, M. C., 1997a, ApJ, accepted

Dove, J. B., Wilms, J., Maisack, M. G., Begelman, M. C., 1997b, ApJ, accepted

Hua, X.-M., Titarchuk, L., 1995, ApJ, 449, 188

Miyamoto, S., Kitamoto, S., Iga, S., et al., 1992, ApJ, 391, L21

Nowak, M. A., Vaughan, B. A., 1996, MNRAS, 280, 227

Nowak, M. A., Vaughan, B. A., Dove, J., Wilms, J., 1996, in D. Wickramasinghe, L. Ferrario, G. Bicknell (eds.), Accretion Phenomena and Related Outflows, IAU Coll. 163, in press

Vaughan, B. A., Nowak, M. A., 1997, ApJ, 474, L43 
